\title{
QUELQUES APPLICATIONS DES FONCTIONELLES ANALYTIQUES
}

\author{
V. Avanissian
}

Résumé. On montre tout d'abord que la transformation $G$ et la convolution d'Hadamard des fonctionnelles analytiques utilisées dans le plan permettent d'obtenir la somme des séries de puissances dont les coefficients sont produits des polynômes orthogonaux clasiques. On applique ensuite la méthode pour obtenir une représentation intégrale des fonctions $p$-harmoniques $h$ de type exponentiel à partir d'une mesure complexe $d \mu$. Pour préciser le support de celle-ci on montre que le type $\tau(h)$ de $h$ calculé à partir de la norme euclidienne est égal au type $\tau_{L}(\tilde{h})$ de $\tilde{h}$ (complexifiée de $h$ ) calculé à partir de la norme de Lie $L$ dans $\mathbf{C}^{N}$, cette étude permet en outre d'affiner certaines inégalités obtenues précédemment.

\section{Introduction}

L'analyse des fonctions génératrices $\mathcal{G}(t, w)(t \in \mathbf{R}, w \in \mathbf{C})$ de certaines classes de polynômes orthogonaux d'une variable fait apparaître la particularité suivante : elles sont holomorphes de $w$ (pour $t$ fixé) hors des points isolés contenus dans $\left.\mathbf{C}_{0}=\mathbf{C} \backslash\right]-\infty, 0$ ] et sont nulles à l'infini. Précisément pour le paramètre $t$ fixé et les convexes compacts adéquats $K \subset \Omega=\{w \in \mathbf{C} \mid-\pi<\operatorname{Im} w<\pi\}$, sont des éléments de l'ensemble $\mathcal{H}_{0}(\Omega(K))$ défini dans $\mathbf{C}^{N}([1], 1.2)$ et qui est ici l'espace des fonctions continues sur $\widetilde{\Omega}(K)=\widetilde{\mathbf{C}} \backslash \exp (-K)$, holomorphe dans $\Omega(K)=\mathbf{C} \backslash \exp (-K)$ et nulle à l'infini. ( $\widetilde{\mathbf{C}}$ étant le compactifié de $\mathbf{C}$ par adjonction du point $\infty)$. Soit $\mathcal{H}^{\prime}(C, K)$ l'espace des fonctionnelles analytiques portables par $K$. La transformation $G: \mathcal{H}^{\prime}(\mathbf{C}, K) \rightarrow \mathcal{H}_{0}(\Omega(K)), T \mapsto G_{T}(w)=$ $\left\langle T_{\zeta},(1 /(1-w \exp \zeta))\right\rangle(c f .[1])$, qui est bijective dans le cas actuel, permet d'associer à chaque $\mathcal{G}(t, w)$ ( $t$ fixé) une fonctionnelle analytique portable par $K$. Les polynômes orthogonaux en $t$ qui apparaissent dans le développement à l'origine de $\mathcal{G}(t, w)$ sont aussi les coefficients de la série $\sum_{n=0}^{\infty} \widehat{T}_{t}(n) w^{n}$ où $T_{t}=G^{-1}[\mathcal{G}(t, w)]$ et $\widehat{T}_{t}(z)$ la transformée de Fourier-Borel de $T_{t}, n$ étant le degré du polynôme $\widehat{T}_{t}(n)$. Cette remarque déjà utilisée par K. Yoshino ( $c f$. [10]) permet d'aborder l'étude de certaines propriétés des fonctions spéciales sous un angle nouveau qui paraît prometteuse. La méthode suivie est susceptible d'être généralisée au cas de plusieurs variables (les résultats dans ce sens seront publiés ultérieurement). La 
première partie de ce travail est consacrée à cette étude. D'autre part la convolution d'Hadamard des fonctionnelles analytiques $(c f .[1], 2.1 .2 ; 2.1 .4)$ permettent de trouver la somme et leur prolongement analytique des séries de puissances dont les coefficients sont le produit des polynômes orthogonaux classiques. Dans la deuxième partie, grâce à des estimations obtenues par complexification, et de l'existence des mesures à support compact, représentant des fonctionnelles analytiques, on donne un énoncé de type Paley-Wiener relatif aux fonctions $p$-harmoniques entières de type exponentiel. Soit $h$ une fonction harmonique (ou $p$-harmonique) dans $\mathbf{R}^{N}$ et $\tilde{h}$ la complexifiée de $h$ dans $\mathbf{C}^{N} ; \tilde{h}$ est une fonction entière dans $\mathbf{C}^{N}$ dont l'ordre de croissance $\rho(h)$ est le même que celui de $h$, mais son type $\tau(\tilde{h})$ est en général diférent de $\tau(h)$ et dépend de la norme choisie. Or pour l'utilisation de la technique d'analyse complexe dans l'étude de certaines propriétés des fonctions harmoniques ou $p$-harmoniques, il est essentiel de savoir si toute constante majorant strictement $\tau(h)$ majeure aussi strictement $\tau(\tilde{h})$. Par exemple il a été établi dans ([2], 6.2.8), en utilisant les inégalités fines sur les polynômes de Legendre que si $|h(x)| \leq a \exp b\|x\|\left(x \in \mathbf{R}^{N}, a, b\right.$ ctes $\left.>0\right)$ alors pour toute $\varepsilon>0$, il existe une constante $A_{\varepsilon}$ telle que

$$
|\tilde{h}(z)| \leq A_{\varepsilon} \exp [(b+\varepsilon)|z|], \quad\left(|z|=\left|z_{1}\right|+\ldots+\left|z_{N}\right|\right) .
$$

La majoration (I) a un inconvénient : pour $z=x$ réel, la norme $|z|$ figurant dans le second membre de (I) ne coïncide pas avec la norme $\|x\|$ qui figure dans l'hypothèse du départ. Or l'utilisation de la norme de Lie $L$ dont la restriction à $\mathbf{R}^{N}$ est la norme euclidienne, permet d'affiner l'inégalité (I) ainsi que d'autres estimations obtenues ailleurs et établir que le type $\tau_{L}(\tilde{h})$ de $\tilde{h}$ calculé à partir de la norme $L$ est égale à $\tau(h)$.

Ces estimations permettent de préciser le support des mesures dans la représentation citée plus haut.

Ce travail a fait l'objet de trois notes aux Comptes Rendus de l'Académie des Sciences [3], [4], [5].

\section{Préliminaires}

Dans la suite on utilisera le diagramme suivant :

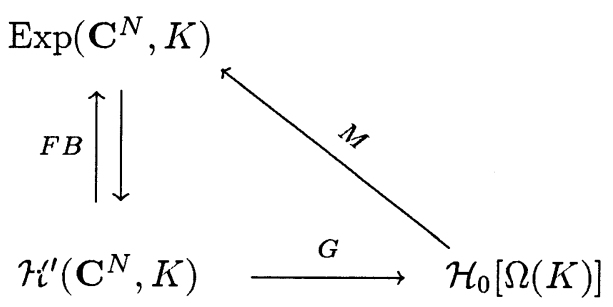

1.1. Commentaire. Soit $\mathcal{H}\left(\mathbf{C}^{N}\right)$ l'espace des fonctions entières sur $\mathbf{C}^{N}$ muni de la topologie de la convergence uniforme sur tout compact. Si $K$ est un 
compact convexe de $\mathbf{C}^{N}$ et $H_{K}(z)=\sup _{\zeta \in K} \operatorname{Re}\left\langle\zeta_{1} z_{1}+\cdots+\zeta_{N} z_{N}\right\rangle$ la fonction d'appui de $K$, on note $: \operatorname{Exp}\left(\mathbf{C}^{N}, K\right)$ l'ensemble des fonctions entières $f$ telles que pour tout $\varepsilon>0$, il existe une constante $M_{\varepsilon}$ vérifiant pour tout $z \in$ $\mathbf{C}^{N}:|f(z)| \leq M_{\varepsilon} \exp \left[H_{K}(z)+\varepsilon|z|\right]$. Remarquons que pour le polydisque fermé $S\left(0, r_{1}, \ldots, r_{N}\right)=S(r)=\left\{z \in \mathbf{C}^{N}|| z_{j} \mid \leq r_{j}, j=1, \ldots, N\right\}$, on a $H_{S}(z)=$ $r_{1}\left|z_{1}\right|+\cdots+r_{N}\left|z_{N}\right|$ et pour $f \in \operatorname{Exp}\left(\mathbf{C}^{N}, S\right),|f(z)| \leq M_{\varepsilon} \exp \left[\left(r_{1}+\varepsilon\right)\left|z_{1}\right|+\right.$ $\left.\cdots+\left(r_{N}+\varepsilon\right) z_{N}\right]$. En particulier $f \in \exp \left(\mathbf{C}^{N},\{0\}\right)$ vérifie pour tout $\varepsilon>0$ : $|f(z)| \leq M_{\varepsilon} \exp (\varepsilon|z|)$. Soit $U=\{z \in \mathbf{C} \mid-\pi<\operatorname{Im} z<\pi\}$ et $\Omega=U^{N}$. Si $K$ est un compact $\subset \Omega$ et $K_{j}$ est sa $j$-ième projection sur $\mathbf{C} \operatorname{par}\left(z_{1}, \ldots, z_{N}\right) \mapsto z_{j}$ on pose :

$$
\Omega(K)=\prod_{j=1}^{N}\left[\mathbf{C} \backslash \exp \left(-K_{j}\right)\right], \quad \widetilde{\Omega}(K)=\prod_{j=1}^{N}\left[\widetilde{\mathbf{C}} \backslash \exp \left(-K_{j}\right)\right]
$$

où $\widetilde{C}$ est le compactifié de $\mathbf{C}$ par adjonction du point $\infty$. L'ensemble des fonctions continues sur $\widetilde{\Omega}(K)$, holomorphes dans $\Omega(K)$ et nulles sur $\widetilde{\Omega}(K) \backslash \Omega(K)$ est noté $\mathcal{H}_{0}[\Omega(K)]=\mathcal{H}_{0}\left[\prod_{j=1}^{N} \mathbf{C} \backslash \exp \left(-K_{j}\right)\right]$.

Le dual topologique de $\mathcal{H}\left(\mathbf{C}^{N}\right)$ est l'espace des fonctionnelles analytiques $\mathcal{H}^{\prime}\left(\mathbf{C}^{N}\right)$. Une forme linéaire $T: \mathcal{H}\left(\mathbf{C}^{N}\right) \rightarrow \mathbf{C}$ est une fonctionnelle analytique si et seulement s'il existe un compact $K \neq \phi$ de $\mathbf{C}^{N}$ et une constante $M_{K}$ tels que pour chaque $f \in \mathcal{H}\left(\mathbf{C}^{N}\right)$,

$$
|\langle T, f\rangle| \leq M_{K} \sup _{z \in K}|f(z)| .
$$

La fonctionnelle analytique $T$ est dite portable par l'ensemble compact $K \subset \mathbf{C}^{N}$ si pour tout voisinage $\omega$ (d'adhérence compacte) de $K$ il existe une constante $M_{\omega} \geq 0$ telle que

$$
|\langle T, f\rangle| \leq M_{\omega} \sup _{z \in \omega}|f(z)| .
$$

La définition implique qu'une fonctionnelle analytique $T$ est portable par au moins un compact de $\mathbf{C}^{N}$ et que si $T$ est portable par le compact $K$ elle est portable par tout compact contenant $K$ et en particulier par l'enveloppe convexe de $K$. Si $T$ est portable par tout compact elle est identiquement nulle. Mais il n'existe pas un plus petit compact portable sauf dans des cas spéciaux. Par exemple :

Proposition (Martineau [9]). Si la fonctionnelle analytique $T$ admet un porteur réel elle admet un plus petit porteur convexe réel.

1.2. Les théorèmes de Hahn-Banach et de F. Riesz impliquent que si $T$ est portable par le compact $K$, pour tout ouvert $\omega \supset K$ il existe une mesure $\mu$ à support compact dans $\omega$ telle que

$$
\langle T, h\rangle=\int h(\zeta) d \mu \quad\left(h \in \mathcal{H}\left(\mathbf{C}^{N}\right)\right) .
$$


La mesure $\mu$ n'est pas unique en général, cependant lorsque $\omega$ est un domaine de Runge, toute mesure représentant $T$ représentera aussi l'unique prolongement de $T$ à $\mathcal{H}(\omega)$ l'espace des fonctions holomorphes dans $\omega$. Si $K$ est un compact de $\Omega=U^{N}$ on note $\mathcal{H}^{\prime}\left(\mathbf{C}^{N}, K\right)$ l'espace vectoriel des fonctionnelles analytiques portables par $K$.

Proposition (Martineau [9]). Soit $T$ une fonctionnelle analytique différente de zéro. Si $T$ est portable par deux convexes compacts $K_{1}, K_{2}$ alors $K_{1} \cap K_{2} \neq \phi$.

1.3. Transformation de Fourier-Borel - (notée $F B)$ - d'une fonctionnelle analytique $T$ est définie par :

$$
z \mapsto \widehat{T}(z)=\left\langle T_{\zeta}, \exp \langle\zeta, z\rangle\right\rangle \quad \text { où }\langle\zeta, z\rangle=\zeta_{1} z_{1}+\cdots+\zeta_{N} z_{N}
$$

on a

$$
\widehat{T}(z)=\sum_{\nu \in \mathbb{N}^{N}} \frac{\left\langle T, \zeta^{\nu}\right\rangle}{\nu !} z^{\nu}, \quad \zeta^{\nu}=\zeta_{1}^{\nu_{1}} \cdots \zeta_{N}^{\nu_{N}}, \quad \nu !=\nu_{1} ! \cdots \nu_{N} !
$$

la convergence étant uniforme sur tout compact. L'énoncé suivant est fondamental.

1.4. Théorème [9]. Soit $T$ une fonctionnelle analytique portable par un convexe compact $K$ de $\mathbf{C}^{N}$. La transformée de Fourier-Borel $\widehat{T}(z)$ de $T$ est une fonction entière élément de $\operatorname{Exp}\left(\mathbf{C}^{N}, K\right)$. Réciproquement soit $f \in \operatorname{Exp}\left(\mathbf{C}^{N}, K\right)$ ( $K$ compact convexe). Il existe une fonctionnelle analytique $T$ portable par $K$ dont la transformée de Fourier-Borel est égale à $f$.

Remarquons que $\widehat{T}(z)=0$ pour tout $z$ implique $T=0$ (cela résulte du développement (2) et du fait que les polynômes sont denses dans $\mathcal{H}\left(\mathbf{C}^{N}\right)$ ).

1.5. Transformation $G(c f .[1])$. Soient $K$ un compact convexe de $\Omega=U^{N}$ et $T \in \mathcal{H}^{\prime}\left(\mathbf{C}^{N}, K\right)$. La transformation $G$ associe à $T$ la fonction :

$$
G_{T}(w)=\left\langle T_{z}, \prod_{j=1}^{N} \frac{1}{1-w_{j} \exp z_{j}}\right\rangle .
$$

1.6. Propriétés (cf. [1]).

a) La fonction $G_{T}(w)$ est holomorphe dans $\Omega(K)=\prod_{j=1}^{N}\left[\mathbf{C} \backslash \exp \left(-K_{j}\right)\right]$ nulle à l'infini. Précisément est un élément de $\mathcal{H}_{0}(\Omega(K))$.

b) La fonction $G_{T}(w)$ est liée à la transformation de Fourier-Borel de $T$ par :

$$
\begin{aligned}
\widehat{T}(z)=( & \left.-\frac{1}{2 i \pi}\right)^{N} \int_{\Gamma_{1} \times \cdots \times \Gamma_{N}} G_{T}(w) \cdot \\
& \cdot \exp \left[-\left(z_{1} \log w_{1}+\cdots+z_{N} \log w_{N}\right)\right] \frac{d w_{1} \ldots d w_{N}}{w_{1} \ldots w_{N}}
\end{aligned}
$$


où $\log w_{j}$ est dans $\left.\left.\mathbf{C}_{0}=\mathbf{C} \backslash\right]-\infty, 0\right]$ la branche vérifiant $\log 1=0 ; \Gamma_{j}$ est pour tout $j$ un contour simple différentiable par morceaux, orienté positivement, contenu dans $\mathbf{C}_{0}$, et entourant $\exp \left(-K_{j}\right)$. D'autre part on a la formule d'inversion

$$
\begin{aligned}
\langle T, h\rangle=(- & \left.\frac{1}{2 i \pi}\right)^{N} \int_{\Gamma_{1} \times \cdots \times \Gamma_{N}} G_{T}(w) . \\
& \cdot h\left(-\log w_{1}, \ldots,-\log w_{N}\right) \frac{d w_{1} \cdots d w_{N}}{w_{1} \ldots w_{N}} .
\end{aligned}
$$

Pour tout $h \in \mathcal{H}\left(\mathbf{C}^{N}\right), T \in \mathcal{H}^{\prime}\left(\mathbf{C}^{N}, K\right) K$ convexe compact de $\Omega=U^{N}$.

La transformation $G: \mathcal{H}^{\prime}\left(\mathbf{C}^{N}, K\right) \rightarrow \mathcal{H}_{0}(\Omega(K)$ ) ( $K$ convexe compact contenu dans $\left.\Omega=U^{N}\right)$ est injective mais en général elle n'est pas bijective. Pourtant si $K=K_{1} \times \cdots \times K_{N}\left(K_{j}\right.$ compact convexe $\left.\subset U\right)$ (et en particulier si $N=$ 1). La transformation $G$ est un isomorphisme de $\mathcal{H}^{\prime}\left(\mathbf{C}^{N}, K\right)$ sur $\mathcal{H}_{0}(\Omega(K))$. L'injectivité de $G$ implique l'égalité $T_{1}=T_{2}$ si $G_{T_{1}}=G_{T_{2}}$.

c) Au voisinage de 0 (respectivement au voisinage de $\infty$ ) on a le développement de Taylor :

$$
G_{T}(w)=\sum_{\nu \in \mathbf{N}^{N}} \widehat{T}(\nu) w^{\nu}
$$

(respectivement

$$
\left.G_{T}(w)=(-1)^{N} \sum_{\substack{\nu \in N^{N} \\ \nu_{j} \geq 1}} \widehat{T}(-\nu) w^{-\nu}\right)
$$

Si $K=S(r) \subset U^{N}$, on a le développement

(7) $G_{T}(w)=(-1)^{\varepsilon\left(w_{1}\right)+\cdots+\varepsilon\left(w_{N}\right)}$.

$$
\text { . } \sum_{\substack{\nu \in N^{N} \\ \nu_{j} \geq \varepsilon\left(w_{j}\right)}} \widehat{T}\left[(-1)^{\varepsilon\left(w_{1}\right)} \nu_{1}, \ldots,(-1)^{\varepsilon\left(w_{N}\right)} \nu_{N}\right] \theta^{\nu_{1}}\left(w_{1}\right) \cdots \theta^{\nu_{N}}\left(w_{N}\right)
$$

où

$$
\varepsilon\left(w_{j}\right)\left(\text { ou } \theta\left(w_{j}\right)\right)= \begin{cases}0\left(\text { ou } w_{j}\right) & \text { si }\left|w_{j}\right|<1 \\ 1\left(\text { ou } w_{j}^{-1}\right) & \text { si }\left|w_{j}\right|>1 .\end{cases}
$$

Le développement (7) étant valable dans $D=\prod_{j=1}^{N}\left(\mathbf{C} \backslash C_{r_{j}}\right)$ avec la convergence uniforme sur tout compact de $D ; C_{r_{j}}$ désigne la couronne :

$$
C_{r_{j}}=\left\{z_{j} \in \mathbf{C}\left|\exp \left(-r_{j}\right) \leq\right| z_{j} \mid \leq \exp r_{j}\right\}
$$

d) Soient $K_{1}, K_{2}$ deux compacts convexes non vides de $\Omega=U^{N}$. L'égalité $T_{1}=T_{2}$ équivaut à $G_{T_{1}}=G_{T_{2}}$. Celle-ci implique $\widehat{T}_{1}(\nu)=\widehat{T}_{2}(\nu)$ pour tout $\nu \in \mathbf{N}^{N}$. Il en résulte qu'une condition nécessaire et suffisante pour que deux fonctionnelles analytiques $T_{1}$ et $T_{2}$ portables par $K_{1}, K_{2}$ respectivement soient égales est que $\widehat{T}_{1}(\nu)=\widehat{T}_{2}(\nu)$ pour tout $\nu \in \mathbf{N}^{N}$. 
1.7. Transformation de Mellin (notée $M$ ). Soient $K$ un compact convexe non vide contenu dans $U^{N}$ et $K_{j}$ la $j$-ième projection de $K$ sur $\mathbf{C}$. La transformation de Mellin associé à chaque élément $\varphi \in \mathcal{H}_{0}[\Omega(K)]$ la fonction :

$$
M(\varphi)(z)=\left(\frac{-1}{2 i \pi}\right)^{N} \int_{\Gamma_{1} \times \cdots \times \Gamma_{N}} \varphi(w) w_{1}^{-z_{1}-1} \cdots w_{N}^{z_{N}-1} d w_{1} \cdots d w_{N}
$$

où $\Gamma_{j}$ est définie dans b). Soit $K=K_{1} \times \cdots \times K_{N}$ : La fonction $M_{\varphi}(z)$ d'après b) est la transformée F.B. d'une fonctionnelle analytique portable par $K$, donc $M_{\varphi}(z) \in \exp \left(\mathbf{C}^{N}, K\right)$.

1.8. Convolution d'Hadamard des fonctionnelles analytiques ( $c f .[1]$ p. 359). Soient $f$ et $g$ deux fonctions analytiques dans le polydisque $S\left(0, r_{1}, \ldots, r_{N}\right)$, $=S(0, r)$ et $\sum_{\nu \in \mathbf{N}^{N}} a_{\nu} z^{\nu}, \sum_{\nu \in \mathbf{N}^{N}} b_{\nu} z^{\nu}$ respectivement le développement en série absolument sommables dans $S(0, r)$ de $f$ et de $g$.

La convolution de $f$ et de $g$ notée $f * g$ est la fonction analytique dans $S(0, r)$ définie par :

$$
(f * g)(z)=\sum_{\nu \in \mathbf{N}^{N}} a_{\nu} b_{\nu} z^{\nu}
$$

on a :

$$
\begin{aligned}
&(f * g)(z)=\left(\frac{1}{2 i \pi}\right)^{N} \int_{\substack{\left|t_{j}\right|=r_{j}^{\prime}<r_{j} \\
1 \leq j \leq N}} f\left(\frac{z_{1}}{t_{1}}, \ldots, \frac{z_{N}}{t_{N}}\right) . \\
& \cdot g\left(t_{1}, \ldots, t_{N}\right) \frac{d t_{1}}{t_{1}} \cdots \frac{d t_{N}}{t_{N}}, \quad\left|z_{j}\right|<r_{j}^{\prime}, \quad 1 \leq j \leq N .
\end{aligned}
$$

La convolution de deux fonctionnelles analytiques $T_{1}$ et $T_{2}$ dans $\mathbf{C}^{N}$ est une fonctionnelle analytique notée $T_{1} * T_{2}$ définie par

$$
\left\langle T_{1} * T_{2}, \Phi\right\rangle=\left\langle T_{1} \otimes T_{2}, \Phi\left(z+z^{\prime}\right)\right\rangle, \quad \Phi \in \mathcal{H}\left(\mathbf{C}^{N}\right)
$$

(cf. [9]) Notons $g_{0}(T)$ le développement $(5)$ de $G_{T}(w)$ au voisinage de 0 .

On a :

1.9. Théorème ([1], p. 360 et 362). Soient $T_{1}$ et $T_{2}$ deux fonctionnelles analytiques portables respectivement par les compacts convexes $K_{1}, K_{2}$ de $\Omega$.

a) $S i K_{1}+K_{2} \subset \Omega$, on a :

$$
g_{0}\left(T_{1} * T_{2}\right)=g_{0}\left(T_{1}\right) * g_{0}\left(T_{2}\right)=\sum_{\nu \in \mathbf{N}^{N}} \widehat{T}_{1}(\nu) \widehat{T}_{2}(\nu) w^{\nu}
$$

pour $\left.w^{\nu}=w_{1}^{\nu_{1}} \cdots \nu_{N}^{\nu_{N}}\right)$.

b) $S$ i $K_{1}+K_{2} \subset \Omega, K_{1}-K_{2}+K_{2} \subset \Omega$ alors 


$$
\begin{aligned}
G_{K_{1}+K_{2}}\left(T_{1} * T_{2}\right)(z)= & \frac{(-1)^{N}}{(2 i \pi)^{N}} \int_{c_{1} \times \cdots \times c_{N}} G_{K_{1}}\left(T_{1}\right)\left(t_{1}, \ldots, t_{N}\right) . \\
& \cdot G_{K_{2}}\left(T_{2}\right)\left(\frac{z_{1}}{t_{1}}, \ldots, \frac{z_{N}}{t_{N}}\right) \frac{d t_{1}}{t_{1}} \cdots \frac{d t_{N}}{t_{N}}
\end{aligned}
$$

pour $z_{j} \notin \exp \left[-\left(D_{j}+p r_{j}\left(K_{2}\right)\right]\right.$ et $1 \leq j \leq N$.

Avec $C_{j}=\exp \left(-c_{j}\right)$ où $c_{j}$ est un polynôme convexe entourant $K_{j}=p_{r_{j}}\left(K_{1}\right)$, contenu dans $U$ et $D_{j}$ est le domaine convexe bordé par $c_{j}$.

\section{Fonctionnelles analytiques liées aux polynômes orthogonaux classiques}

2.1. Plaçons-nous dans le plan complexe et considérons respectivement les fonctions génératrices des cinq classes de polynômes suivants : Laguerre $L_{n}^{\alpha}(t)$ (d'ordre entier $\alpha \geq 0$ ), Chebyshev $C_{n}(t)$ de $1^{\text {re }}$ espèce, Chebyschev $U_{n}(t)$ de $2^{\text {ième }}$ espèce, Legendre $\widetilde{L}_{n}(t)$, Gegenbauer $C_{n}^{\lambda}(t)(c f .[7]$ p. 1-97)

$$
\left\{\begin{array}{c}
\mathcal{G}_{L^{\alpha}}(t, w)=(1-w)^{-\alpha-1} \exp (-t w / 1-w)=\sum_{n=0}^{\infty} L_{n}^{\alpha}(t) w^{n} \\
\quad(t \in \mathbf{R},|w|<1) \\
\mathcal{G}^{(1)}(t, w)=(1-t w)\left(1-2 t w+w^{2}\right)^{-1}=\sum_{n=0}^{\infty} C_{n}(t) w^{n} \\
\mathcal{G}^{(2)}(t, w)=\left(1-2 t w+w^{2}\right)^{-1}=\sum_{n=0}^{\infty} U_{n}(t) w^{n} \\
\mathcal{G}_{\widetilde{L}}(t, w)=\left(1-2 t w+w^{2}\right)^{-1 / 2}=\sum_{n=0}^{\infty} \widetilde{L}_{n}(t) w^{n}, \quad\left[\mathcal{G}_{\widetilde{L}}(t, 0)=1\right] \\
\mathcal{G}^{\lambda}(t, w)=\left(1-2 t w+w^{2}\right)^{-\lambda}=\sum_{n=0}^{\infty} C_{n}^{\lambda}(t) w^{n}, \quad\left[g^{\lambda}(t, 0)=1\right] \\
\quad(|t|<1,|w|<1, \lambda>0) .
\end{array}\right.
$$

Pour $t$ fixé, $\mathcal{G}_{L^{\alpha}} \in \mathcal{H}_{0}(\mathbf{C} \backslash \exp \{0\})=\mathcal{H}_{0}(\mathbf{C} \backslash\{1\})$.

Les autres appartiennent à $\mathcal{H}_{0}[\mathbf{C} \backslash \exp (-I(t))]$ pour $|t|<1$ où $I(t)=$ $i\left[-\theta_{t}, \theta_{t}\right], \cos \theta_{t}=t, 0<\theta_{t}<\pi$.

L'application $G^{-1}$ permet d'associer à chacune de ces fonctions génératrices une fonctionnelle analytique. Soient :

$$
\begin{aligned}
T_{t}^{L^{\alpha}} & =G^{-1}\left(\mathcal{G}_{L^{\alpha}}\right), \quad T_{t}^{1}=G^{-1}\left(\mathcal{G}^{(1)}\right), \\
T_{t}^{2} & =G^{-1}\left(\mathcal{G}^{(2)}\right), \quad T_{t}^{\widetilde{L}}=G^{-1}\left(\mathcal{G}_{\widetilde{L}}\right), \quad T_{t}^{\lambda}=G^{-1}\left(\mathcal{G}^{\lambda}\right) .
\end{aligned}
$$

Quel que soit le paramètre $t, T_{t}^{L^{a}}$ est portable par $\{0\}$, les autres sont portables par $I(t) \subset i]-\pi, \pi[$. On remarquera que l'image de $I(t)$ par l'application $z \mapsto \exp (-z)$ est l'arc du cercle unité situé dans $\mathbf{C}_{0}$ limité aux points singuliers communs des fonctions génératrices ci-dessus. En comparant chaque série de (12) avec le développement (5) où $T=T_{t}$, est la fonctionnelle analytique associée à la 
fonction génératrice de la série, on constate que les polynômes orthogonaux figurant dans la série envisagée sont de la forme $\widehat{T}_{t}(n)$. Cela permet d'aborder l'étude de certaines propriétés des fonctions spéciales sous un angle nouveau qui paraît prometteur (voir aussi [10]). La méthode suivie est en outre susceptible d'être utilisée dans le cas de plusieurs variables. D'autre part la particularité des compacts portables des fonctionnelles (13) conduit à profiter du théorème 1.9.

2.2. Théorème 1. Pour tout $\alpha \geq 0, \alpha \in \mathbf{N}$, il existe une fonctionnelle analytique $T_{t}^{\alpha}$ dépendant d'un paramètre $t \in \mathbf{R}$ et portable par $\{0\}$ telle que pour tout $n \in \mathbf{N}, \widehat{T}_{t}^{\alpha}(n)=L_{n}^{\alpha}(t)$. Le prolongement analytique $L_{z}^{\alpha}(t)$ des polynômes de Laguerre $L_{n}^{\alpha}(t)$ pour $t$ fixé, est la fonction entière de type exponentiel nul $\widehat{T}_{t}^{\alpha}(z)$. Pour $|t|<1$, à toute autre classe (S) de polynômes $P_{n}(t)$ de (12) correspond une fonctionnelle analytique $T_{t}^{(S)}$ dépendant de $t$ et portable par $I(t)$ telle que pour tout $n, \widehat{T}_{t}^{(S)}(n)=P_{n}(t)$. Tout polynôme $P_{n}(t)$ de $(S)$ est pour $t$ fixé, la restriction à $z=n$ de la fonction entière $\widehat{T}_{t}^{(S)}(z)$ de type exponentiel $<\pi$.

La dernière affirmation résulte du fait que la fonction d'appui $H_{I(t)}(z)$ de $I(t)=\left[-i \theta_{t}, i \theta_{t}\right]\left(0<\theta_{t}<\pi\right), \cos \theta_{t}=t$, est $\theta_{t}|\operatorname{Im} z| \leq \theta_{t} \pi$ et que la transformation de Fourier-Borel $\widehat{T}_{t}^{(S)}(z)$ vérifie pour tout $\varepsilon$, avec $\varepsilon+\theta_{t}<\pi$ :

$$
\left|\widehat{T}_{t}^{(S)}(z)\right| \leq A_{\varepsilon} \exp \left[\theta_{t}|\operatorname{Im} z|+\varepsilon|z|\right] \leq A_{\varepsilon} \exp \left[\left(\varepsilon+\theta_{t}\right)\right]|z| \leq A_{\varepsilon} \exp \pi|z|
$$

$A_{\varepsilon}$ étant une constante dépendant de $\varepsilon$.

2.3. Proposition. On a pour tout $N \geq 1$ :

$$
\begin{aligned}
& \sum_{n=0}^{\infty} L_{n}^{\alpha_{1}}\left(t_{1}\right) \cdots L_{n}^{\alpha_{N}}\left(t_{N}\right) z^{N}= \\
& \left(-\frac{1}{2 i \pi}\right)^{N} \int_{\substack{\left|w_{j}-1\right|=r_{j} \\
1 \leq j \leq N}} \frac{\exp \left[-\sum_{j=1}^{N} t_{j} w_{j} / 1-w_{j}\right]}{\left(w_{1} w_{2} \ldots w_{N}-z\right) \prod_{j=1}^{N}\left(1-w_{j}\right)^{\alpha_{j}+1}} d w_{1} \cdots d w_{N} \\
& |z| \leq r<1, r_{j} \leq 1-(r+\varepsilon)^{1 / N}, t \in \mathbf{R}^{* N}, \alpha \in \mathbf{N}^{N}, 0<r+\varepsilon<1 .
\end{aligned}
$$

Démonstration. La fonction

$$
\prod_{j=1}^{N} \frac{\exp \left[-\frac{t_{j} w_{j}}{1-w_{j}}\right]}{\left(1-w_{j}\right)^{\alpha_{j}+1}}=\prod_{j=1}^{N} \mathcal{G}_{L^{\alpha_{j}}}\left(t_{j}, w_{j}\right)
$$

est un élément de $\mathcal{H}_{0}[\Omega(\{0\})]=\mathcal{H}_{0}\left[\prod_{j=1}^{N}(\mathbf{C} \backslash\{1\})\right]$. Soit

$$
T_{t}^{\alpha}=G^{-1}\left(\prod_{j=1}^{N} \mathcal{G}_{L^{\alpha_{j}}}\left(t_{j}, w_{j}\right)\right)
$$


d'après (3) on a

$$
\begin{aligned}
& \widehat{T}_{t}^{\alpha}(z)= \\
& \left(-\frac{1}{2 i \pi}\right)^{N} \int_{\Gamma_{1} \times \cdots \times \Gamma_{N}} \frac{\exp \left[-\sum_{j=1}^{N} \frac{t_{j} w_{j}}{1-w_{j}}\right]}{\left(1-w_{1}\right)^{\alpha_{1}} \cdots\left(1-w_{N}\right)^{\alpha_{N}}} \frac{d w_{1} \cdots d w_{N}}{w_{1}^{z_{1}+1} \cdots w_{N}^{z_{N}+1}}
\end{aligned}
$$

où $\Gamma_{j}$ est pour tout $j$ un chemin fermé dans $\mathbf{C}_{0}$ d'orientation positive, d'indice 1 par rapport au point $w_{j}=1$ et $w_{j}^{z_{j}+1}=\left(z_{j}+1\right) \exp \log w_{j}$ avec la branche de logarithme nulle au point $w_{j}=1$. D'autre part,

$$
\widehat{T}_{t}^{\alpha}(z)=\prod_{=1}^{N}\left(-\frac{1}{2 i \pi} \int_{\Gamma_{j}} \frac{\exp \left[\left(-t_{j} w_{j}\right)\left(1-w_{j}\right)^{-1}\right] d w_{j}}{\left(1-w_{j}\right)^{\alpha_{j}} w_{j}^{z_{j}+1}}\right)
$$

et

$$
\widehat{T}_{t}^{\alpha}(\nu)=\prod_{j=1}^{N}\left(-\frac{1}{2 i \pi} \int_{\Gamma_{j}} \frac{\exp \left[\left(-t_{j} w_{j}\right)\left(1-w_{j}\right)^{-1}\right] d w_{j}}{\left(1-w_{j}\right)^{\alpha_{j}} w_{j}^{v_{j}+1}}\right)
$$

pour $\nu=\left(\nu_{1}, \ldots, \nu_{N}\right)$.

Le changement de variable $u_{j}=t_{j} /\left(1-w_{j}\right), j=1, \ldots, N$ et un calcul simple de résidus montre que

$$
\widehat{T}_{t}^{\alpha}(\nu)=\prod_{j=1}^{N} \frac{e^{t_{j}} t_{j}^{-\alpha_{j}}}{\nu_{j} !}\left(\frac{\partial}{\partial t_{j}}\right)^{\nu_{j}}\left(e^{-t_{j}} t_{j}^{\nu+\alpha_{j}}\right)=\prod_{j=1}^{N} L_{\nu_{j}}^{\alpha_{j}}\left(t_{j}\right)
$$

d'après la formule de Rodrigues ( $c f$. [7] p. 76).

Donc, d'après (16) et (17) où $\nu_{j}=n, j=1, \ldots, N$, on a :

$$
\begin{aligned}
L_{n}^{\alpha_{1}}\left(t_{1}\right) \cdots & L_{n}^{\alpha_{N}}\left(t_{N}\right) z^{n}=\widehat{T}_{t}^{\alpha}(\nu) z^{n}= \\
= & \left(-\frac{1}{2 i \pi}\right)^{N} \int_{\Gamma_{1} \times \cdots \times \Gamma_{N}} \frac{\exp \left[-\sum_{j=1}^{N} t_{j} w_{j}\left(1-w_{j}\right)^{-1}\right]}{\left(1-w_{1}\right)^{\alpha_{j}} \cdots\left(1-w_{N}\right)^{\alpha_{N}}} \\
& \cdot\left(\frac{z}{w_{1} \ldots w_{N}}\right)^{n} \frac{d w_{1} \cdots d w_{N}}{w_{1} \cdots w_{N}}
\end{aligned}
$$

Pour $|z| \leq r<1$ et $\varepsilon>0$ tel que $r+\varepsilon<1$, choisissons dans (18) $\Gamma_{j}=$ $\left\{w_{j}|| w_{j}-1 \mid=r_{j} \leq 1-(r+\varepsilon)^{1 / N}\right\}$. Alors, pour $w_{j} \in \Gamma_{j}(1 \leq j \leq N)$,

$$
\begin{aligned}
\left|w_{1} \cdots w_{N}\right| & \geq\left(1-\left|w_{1}-1\right|\right) \cdots\left(1-\left|w_{N}-1\right|\right) \\
& \geq\left(1-r_{1}\right) \cdots\left(1-r_{N}\right)>r+\varepsilon
\end{aligned}
$$

et

$$
|z| /\left|w_{1} \ldots w_{N}\right|<1, \quad \frac{1}{w_{1} \cdots w_{N}} \sum_{n=0}^{\infty}\left(\frac{z}{w_{1} \cdots w_{N}}\right)^{n}=\frac{1}{w_{1} \cdots w_{N}-z} .
$$

D'où l'égalité (14).

En particulier le changement de variable ci-dessus conduit à : 
2.4. Corollaire. Pour tout $N \geq 1$ on a :

$$
\begin{aligned}
e^{-[t]} & \sum_{n=0}^{\infty} L_{n}^{0}\left(t_{1}\right) \cdots L_{n}^{0}\left(t_{N}\right) z^{n} \\
& =\left(\frac{1}{2 i \pi}\right)^{N} \int_{\substack{\left|u_{j}\right|=\frac{\left|t_{j}\right|}{r_{j}} \\
1 \leq j \leq N}} \frac{\exp (-[u]) d u_{1} \cdots d u_{N}}{\left(u_{1}-t_{1}\right) \cdots\left(u_{N}-t_{N}\right)-u_{1} u_{2} \cdots u_{N} z} \\
r_{j} & =1-(r+\varepsilon)^{1 / N}, \quad|z|<r+\varepsilon<1, \quad[u]=u_{1}+\cdots+u_{N}, \quad t \neq 0 .
\end{aligned}
$$

\section{Applications I}

3.1. Le théorème 1.9. permet d'obtenir une représentation intégrale du prolongement analytique hors du disque de convergence de certaines séries de puissances dont les coefficients sont le produit des polynômes dont les fonctionnelles analytiques associées sont définies au 2.1 et qui sont portables par les compacts $K=i\left[-\theta_{t}, \theta_{t}\right], \cos \theta_{t}=t, 0 \leq \theta_{t}<\pi$ à l'exception de $T_{t}^{L^{\alpha}}$ qui est pour tout $t \in \mathbf{R}$, portable par $\{0\}$.

Dans le théorême 1.9 afin de réaliser les conditions sur les portables : $K_{1}+$ $K_{2} \subset \Omega, K_{1}-K_{1}+K_{2} \subset \Omega$ on supposera sauf mention de contraire que $\left|\theta_{t}\right|<\pi / 3$, c'est-à-dire $1 / 2<\left|\cos \theta_{t}\right|=|t|<1$. Pour le polygone convexe (c) entourant $K$, on choisira un rectangle $c_{t}(\varepsilon)$ de largeur $2 \varepsilon$ et de longueur $2\left(\theta_{t}+\varepsilon\right)$ centré en 0 , $\varepsilon$ étant arbitrairement petit. Soit $\mathcal{R}_{t}(\varepsilon) \subset \Omega=U$ le domaine convexe bordé par $c_{t}(\varepsilon)$. L'image par l'application $\underline{\ell}: z \mapsto e^{-z}$ de $\mathcal{R}_{t}(\varepsilon)$ est le domaine défini par $\underline{\ell}\left(\mathcal{R}_{t}(\varepsilon)\right):\left\{w \in \mathbf{C}\left|e^{-\varepsilon}<\right| w \mid<e^{\varepsilon},-\theta_{t}-\varepsilon<\operatorname{Arg} w<\theta_{t}+\varepsilon\right\}$. On remarquera que ce domaine est un voisinage de l'arc $\Gamma_{t}$ du cercle unité $|w|=1$, limité aux points $e^{ \pm i \theta_{t}}$ (l'image de $i\left[-\theta_{t}, \theta_{t}\right]$ par $\underline{\ell}$ ). Réciproquement quel que soit le contour simple frontière d'un $\varepsilon$ voisinage $V$ de $\underline{\ell}\left(i\left[-\theta_{t}, \theta_{t}\right]\right)$, il existe un $\varepsilon>0$ tel que $\underline{\ell}\left(\mathcal{R}_{t}(\varepsilon)\right) \subset V$.
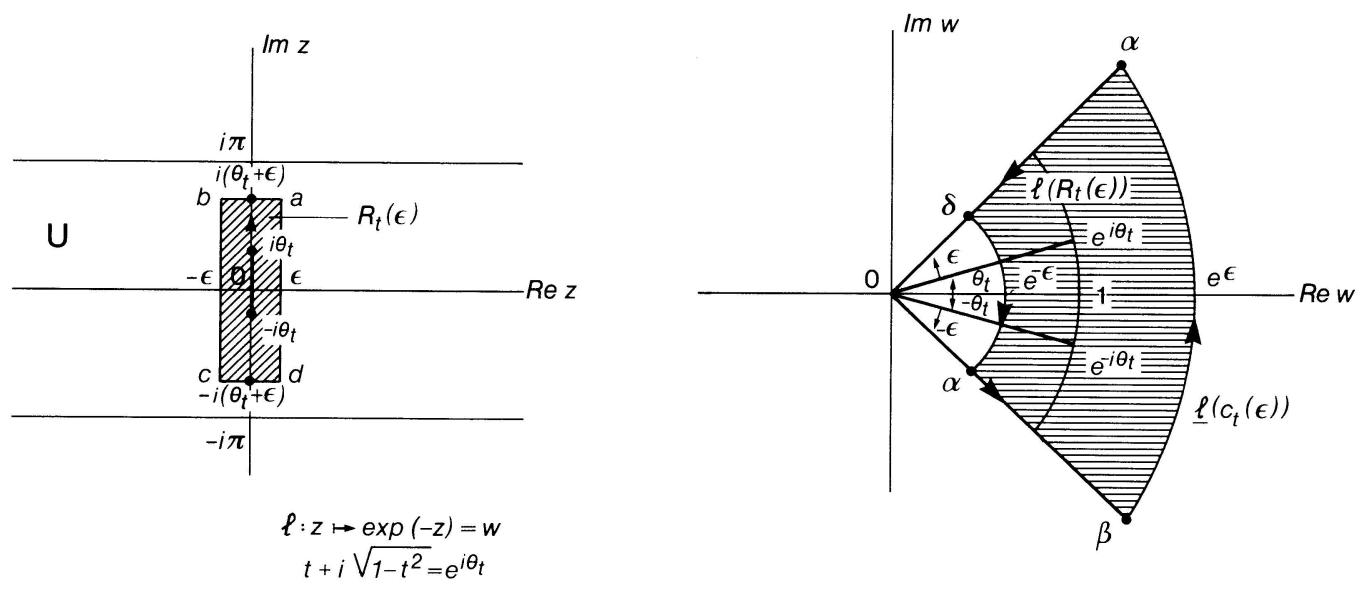
3.2. Théorème. Soient $P_{n}(t)$ [respectivement $\left.Q_{n}(t)\right], n=0,1,2, \ldots$, une suite de polynômes appartenant à l'une des classes de $(12), \mathcal{G}_{p}$ (respectivement $\mathcal{G}_{Q}$ ) la fonction génératrice des $P_{n}$ ( respectivement $Q_{n}$ ) et

$$
S_{t}(z)=\sum_{n=0}^{\infty} P_{n}\left(t_{1}\right) Q_{n}\left(t_{2}\right) z^{n} \quad(|z|<1)
$$

avec $\frac{1}{2}<\left|t_{1}\right|<1, \frac{1}{2}<\left|t_{2}\right|<1$ ou bien $t_{1} \in \mathbf{R}$ (respectivement $t_{2} \in \mathbf{R}$ ) si $P_{n}$ (respectivement $Q_{n}$ ) est égal à $L_{n}^{\alpha}(t)$ :

A. Si $P_{n}=L_{n}^{\alpha}(n \in \mathbf{N})$, pour tout $\varepsilon$-voisinage $V_{\varepsilon}\left(t_{2}\right)$ de $\exp \left(-i\left[-\theta_{2}, \theta_{2}\right]\right)$ $\left(\cos \theta_{2}=t_{2}, 0 \leq \theta_{2}<\pi\right)$ de frontière $\Gamma_{t_{2}}$, la somme $S_{t}(z)$ pour $t$ fixé a un prolongement holomorphe dans tout le domaine $\mathbf{C} \backslash V_{\varepsilon}\left(t_{2}\right)$. Le prolongement est donné par

$$
\widetilde{S}_{t}(z)=-\frac{1}{2 i \pi} \int_{\Gamma_{t_{2}}} \mathcal{G}_{L^{\alpha}}\left(t_{1}, w\right) \mathcal{G}_{Q}\left(t_{2}, \frac{z}{w}\right) \frac{d w}{w}
$$

qui peut être calculé par la méthode des résidus.

En particulier pour $|z|<1, t=\left(t_{1}, t_{2}\right) \in \mathbf{R}$ :

$$
\begin{aligned}
& \sum_{n=0}^{\infty} L_{n}^{\alpha}\left(t_{1}\right) L_{n}^{\beta}\left(t_{2}\right) z^{n}= \\
& \quad=\operatorname{Rés}\left[\frac{\exp \left(-t_{1} w /(1-w)\right) \exp \left(-t_{2} z /(w-z)\right) w^{\beta}}{(1-w)^{\alpha+1}(w-z)^{\beta+1}}\right]_{w=z} .
\end{aligned}
$$

B. Dans tous les autres cas si $V_{\varepsilon}(t)$ est un $\varepsilon$-voisinage de frontière $\Gamma_{t}$ de $\exp \left(-i\left[-2 \theta_{t}, 2 \theta_{t}\right]\right), 0<\theta_{t}=\max \left(\theta_{t_{1}}, \theta_{t_{2}}\right)<\pi / 3$ et $\frac{1}{2}<\left|t_{j}\right|<1, j=1,2$, la somme de la série (20) pour $t$ fixé se prolonge holomorphiquement dans tout le domaine $\mathbf{C} \backslash V_{\varepsilon}(t)$. Le prolongement est donné par l'intégrale $-1 / 2 i \pi \int_{\Gamma_{t}} \mathcal{G}_{P}\left(t_{1}, w\right)$. $\mathcal{G}_{Q}\left(t_{2}, z / w\right) d w / w$ qui peut être calculée par la méthode des résidus.

\section{Applications II}

3.3. Soit $U_{n}(t), n=0,1,2, \ldots$, la suite de polynômes de $2^{\text {ième }}$ espèce de Chebyshev. On a

$$
\sum_{n=0}^{\infty} U_{n}^{2}(t) z^{n}=\frac{1}{1-z} \frac{1+z}{z^{2}-2\left(2 t^{2}-1\right) z+1} \quad\left(\frac{1}{2}<|t|<1,|z|<1\right) .
$$

En effet, soit $V_{\varepsilon}(t)$ un $\varepsilon$-voisinage de $\ell\left(i\left[-2 \theta_{t}, 2 \theta_{t}\right]\right)$ avec $\cos \theta_{t}=t\left(0 \leq \theta_{t}<\pi / 3\right)$ la somme $S\left(U_{n}^{2}, t, z\right)$ de la série (23) est égale d'après (21) à

$$
\begin{aligned}
S\left(U_{n}^{2}, t, z\right) & =-\frac{1}{2 i \pi} \int_{\Gamma_{\varepsilon}(t)} \frac{1}{1-2 t w+w^{2}} \frac{w}{w^{2}-2 t z+z^{2}} d w \\
& =-\frac{1}{2 i \pi} \int \frac{w d w}{\left(w-e^{i \theta_{t}}\right)\left(w-e^{-i \theta_{t}}\right)\left(w-z e^{i \theta_{t}}\right)\left(w-z e^{-i \theta}\right)} .
\end{aligned}
$$


La.fonction $F$ sous signe d'intégrale a quatre pôles qui sont :

$$
e^{i \theta_{t}}, \quad e^{-i \theta_{t}}, \quad z e^{i \theta_{t}}, \quad z e^{-i \theta_{t}}
$$

en choisissant pour $\Gamma_{\varepsilon}(t)$ un contour convenable on peut supposer que les deux derniers pôles sont en dehors de $V_{\varepsilon}(t)$ et en remarquant que $\lim _{R \rightarrow \infty} \int_{|w|=R} F d w=0$ la méthode des résidus donne :

$$
S\left(U_{n}^{2}, t, z\right)=\operatorname{Res}[F]_{w=z e^{i \theta_{t}}}+\operatorname{Res}[F]_{w=z e^{-i \theta_{t}}} .
$$

Le premier résidus est égal à

$$
\frac{z e^{i \theta_{t}}}{\left(z e^{i \theta_{t}}-e^{i \theta_{t}}\right)\left(z e^{i \theta_{t}}-e^{-i \theta_{t}}\right)\left(z e^{i \theta_{t}}-z e^{-i \theta_{t}}\right)}=\frac{1}{2 i} \frac{1}{(z-1)\left(z e^{i \theta_{t}}-e^{-i \theta_{t}}\right) \sin \theta_{t}} .
$$

Le second résidu est égal à

$$
-\frac{1}{2 i} \frac{1}{(z-1)\left(z e^{-i \theta_{t}}-e^{i \theta_{t}}\right) \sin \theta_{t}} .
$$

D'où

$$
\begin{aligned}
S\left(U_{n}^{2}, t, z\right) & =-\frac{1}{2 i} \frac{1}{(z-1) \sin \theta_{t}}\left(\frac{1}{z e^{\theta_{t}}-e^{-i \theta_{t}}}-\frac{1}{z e^{-i \theta_{t}}-e^{i \theta_{t}}}\right) \\
& =\frac{-1}{2 i(z-1) \sin \theta_{t}} \frac{z e^{-i \theta_{t}}-e^{i \theta_{t}}-z e^{i \theta_{t}}+e^{-i \theta_{t}}}{z^{2}-z\left(e^{2 i \theta_{t}}+e^{-2 i \theta_{t}}\right)+1} \\
& =\frac{1}{2 i(z-1) \sin \theta_{t}} \frac{z\left(e^{i \theta_{t}}-e^{-i \theta_{t}}\right)+\left(e^{i \theta_{t}}-e^{-i \theta_{t}}\right)}{z^{2}-2 z \cos 2 \theta_{t}+1} \\
& =\frac{1}{2 i(z-1) \sin \theta_{t}} \frac{(z+1)\left(e^{i \theta_{t}}-e^{-i \theta_{t}}\right)}{z^{2}-2 z \cos 2 \theta_{t}+1} \\
& =\frac{z+1}{z-1} \frac{1}{z^{2}-2 z \cos \theta_{t}+1} .
\end{aligned}
$$

Finalement

$$
\sum_{n=0}^{\infty} U_{n}^{2}(t) z^{n}=\frac{1}{1-z} \frac{1+z}{z^{2}-2\left(2 t^{2}-1\right) z+1} \quad\left(\frac{1}{2}<|t|<1,|z|<1\right) .
$$

Le second membre de (23) définit le prolongement analytique du premier membre dans $D_{\varepsilon}^{t}=\mathbf{C} \backslash V_{\varepsilon}(t)$ ( $\varepsilon$ arbitraire).

3.4. Remarque. La singularité $z=1$ au second membre de (23) est naturelle puisque les coefficients de la série (23) sont tous non négatifs. 
Quelques applications des fonctionnelles analytiques

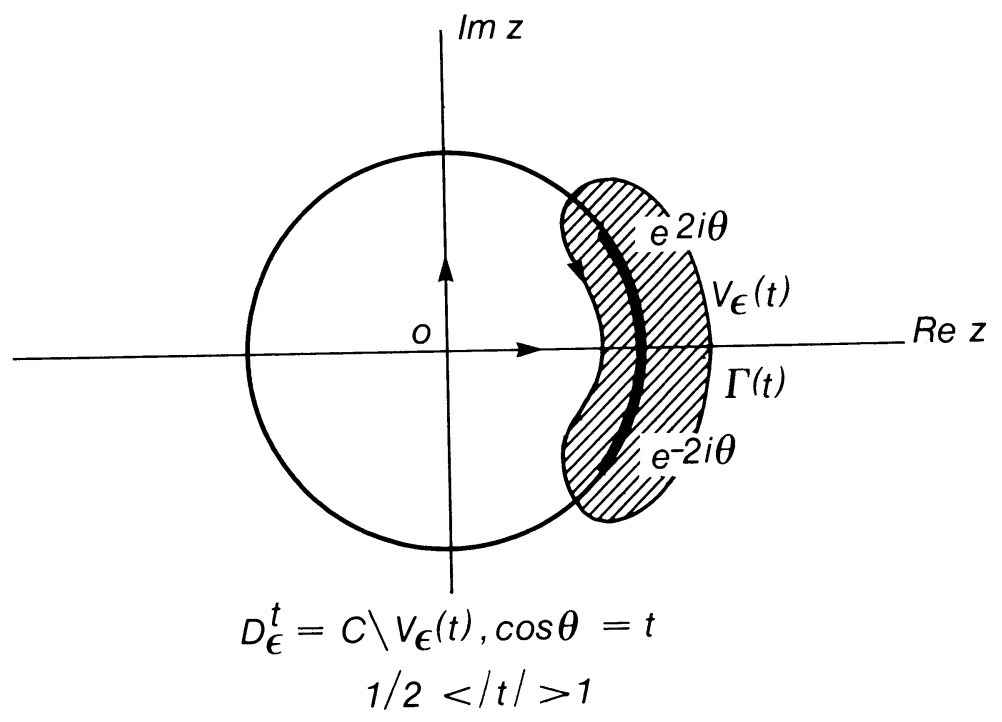

Il est bien connu que $U_{n}(\cos \theta)=(\sin (n+1) \theta / \sin \theta), \theta \neq 2 k \pi$. On déduit de (23) :

$$
\sum_{n=0}^{\infty}\left[\frac{\sin (n+1) \theta}{\sin \theta}\right]^{2} z^{n}=\frac{1}{1-z} \frac{1+z}{z^{2}-2 \cos 2 \theta z+1}
$$

pour $|\theta|<\frac{\pi}{3},|z|<1$.

3.5. Calcul de $\sum_{n=0}^{\infty} C_{n}^{2}(t) z^{n}$.

$$
S\left(C_{n}^{2}, t, z\right)=\sum_{n=0}^{\infty} C_{n}^{2}(t) z^{n}=-\frac{1}{2 i \pi} \int_{\Gamma_{t}} \frac{1-t w}{1-2 t w+w 2} \frac{w-t z}{w^{2}-2 t z w+z^{2}} d w
$$

La méthode des résidus conduit à :

$$
S\left(C_{n}^{2}, t, z\right)=\operatorname{Rés}\left[F_{1}\right]_{w=z e^{i}} i \theta_{t}+\operatorname{Rés}\left[F_{1}\right]_{w=z e^{-i \theta_{t}}}
$$

où $F_{1}$ est la fonction sous l'intégrale dans (25):

$$
F_{1}=\frac{(1-t w)(w-t z)}{\left(w-e^{i \theta_{t}}\right)\left(w-e^{-i \theta_{t}}\right)\left(w-z e^{i \theta_{t}}\right)\left(w-z e^{-i \theta_{t}}\right)} .
$$

On obtient

$$
S\left(C_{n}^{2}, t, z\right)=\frac{1}{1-z} \frac{t^{2} z^{2}-\left(3 t^{2}-1\right) z+1}{z^{2}-2\left(2 t^{2}-1\right) z+1}
$$

avec $\frac{1}{2}<|t|<1,|z|<1$. 
Remarque. On sait que $C_{n}(\cos \theta)=\cos n \theta$. Donc,

$$
\sum_{n=0}^{\infty} \cos ^{2} n \theta \cdot z^{n}=\frac{1}{1-z} \frac{z^{2} \cos ^{2} \theta-\left(\cos 2 \theta+\cos ^{2} \theta\right) z+1}{z^{2}-2 z \cos 2 \theta+1},
$$

avec $|\theta|<\pi / 3,|z|<1$, évidemment (26) peut être obtenue directement par un calcul très élémentaire et pour tout $\theta$. Le second membre de (26) définit le prolongement analytique dans $D_{\varepsilon}^{t}=\mathbf{C} \backslash V_{\varepsilon}(t)$ de la somme de la série du $1^{\text {re }}$ membre.

3.6. De même en procédant comme ci-dessus on obtient :

$$
S\left(C_{n}, U_{n}, t, z\right)=\sum_{n=0}^{\infty} C_{n}(t) U_{n}(t) z^{n}=\frac{1}{1-z} \frac{1-\left(2 t^{2}-1\right) z}{z^{2}-2\left(2 t^{2}-1\right) z+1}
$$

pour $\frac{1}{2}<|t|<1,|z|<1$ et

$$
\sum_{n=0}^{\infty} \cos n \theta \frac{\sin (n+1) \theta}{\sin \theta} z^{n}=\frac{1}{1-z} \frac{1-z \cos 2 \theta}{z^{2}-2 z \cos 2 \theta+1}
$$

pour $|\theta|<\pi / 3,|z|<1$.

Le second membre de (29) est le prolongement analytique de la somme de la série de $1^{\text {re }}$ membre dans $D_{\varepsilon}^{t}=\mathbf{C} \backslash V_{\varepsilon}(t)$.

3.7. De même pour $\frac{1}{2}<t<1,|z|<1$ la méthode fournit la somme des séries suivantes sous la forme $\operatorname{Rés}[F]_{w=z e^{i \theta_{t}}}+\operatorname{Rés}[F]_{w=z e^{-i \theta_{t}}}$ où $F$ désigne dans chaque cas la fonction sous signe de l'intégrale :

$$
\begin{gathered}
\sum_{n=0}^{\infty} \widetilde{L}_{n}(t) U_{n}(t) z^{n}=-\frac{1}{2 i \pi} \int_{\Gamma_{t}} w\left(w^{2}-2 t w+1\right)^{-1 / 2}\left(w^{2}-2 t z w+z^{2}\right)^{-1} d w \\
\sum_{n=0}^{\infty}\left[\widetilde{L}_{n}(t)\right]^{2} z^{n}=-\frac{1}{2 i \pi} \int_{\Gamma_{t}}\left(w^{2}-2 t w+1\right)^{-1 / 2}\left(w^{2}-2 t z w+z^{2}\right)^{-1 / 2} d w \\
\sum_{n=0}^{\infty} \widetilde{L}_{n}(t) C_{n}(t) z^{n}=-\frac{1}{2 i \pi} \int_{\Gamma_{t}}(w-t z)\left(w^{2}-2 t w+1\right)^{-1 / 2}\left(w^{2}-2 t z w+z^{2}\right)^{-1} d w
\end{gathered}
$$

\section{Une représentation intégrale des fonctions $p$-harmoniques de type exponentiel}

4.1. Une fonction réelle $f$ est dite $p$-harmonique dans un ouvert $\Omega \subset \mathbf{R}^{N}$ s'il existe un plus petit entier $p$, tel que $f \in \mathcal{C}^{2 p}(\Omega)$ et $\Delta^{p} f=0, \Delta$ étant l'opérateur laplacien itéré $p$-fois. L'énoncé suivant est à rapprocher d'un théorème de PaleyWiener. 
4.2. Théorème. Soit $f$ une fonction $p$-harmonique $(p \geq 1)$ dans tout $\mathbf{R}^{N}$ vérifiant :

$$
|f(x)| \leq a e^{b\|x\|} \quad\left(x \in \mathbf{R}^{N}, a, b \text { constantes }>0\right)
$$

$\|x\|=\left(x_{1}^{2}+\cdots+x_{N}^{2}\right)^{1 / 2}$. Alors pour tout $\varepsilon>0$ il existe une mesure $d \mu_{f, \varepsilon}$ dans $\mathbf{C}^{N}$ à support compact contenu dans le polydisque $C_{b, \varepsilon}=\left\{z \in \mathbf{C}^{N}|| z_{j} \mid<b+\varepsilon\right.$, $j=1, \ldots, N\}$ et de masse totale égale à $f(0)$ telle que

$$
\begin{aligned}
f(x) & =\int_{\mathbf{C}^{N}} e^{\langle x, \zeta\rangle} d \mu_{f, \varepsilon}(\zeta) \quad\left(x \in \mathbf{R}^{N}\right) \\
\langle x, \zeta\rangle & =x_{1} \zeta_{1}+\cdots+x_{N} \zeta_{N} .
\end{aligned}
$$

La démonstration du théorème utilise la complexification $\tilde{f}$ de $f(c f .[2]$, p. 139). La majoration (37) (qui sera établie plus loin) montre que $\tilde{f}$ est la transformée de Fourier-Borel $\widehat{T}(z)$ d'une fonctionnelle analytique $T$ portable par le compact $K=\left\{z \in \mathbf{C}^{N}|| z_{j} \mid \leq b, j=1, \ldots, N\right\}$. Soit $d \mu_{f, \varepsilon}$ une mesure représentant $T$ à support compact contenu dans $C_{b, \varepsilon}$. On a alors (cf. 1.2)

$$
\tilde{f}(z)=\widehat{T}(z)=\int e^{\langle z, \zeta\rangle} d \mu_{f, \varepsilon}(\zeta)
$$

pour $z$ réel on obtient (31).

4.3. Afin de préciser le support de la mesure figurant dans le théorème 4.2. on va faire une étude préliminaire en comparant l'ordre et le type de croissance d'une fonction $p$-harmonique $h$ dans tout $\mathbf{R}^{N}$, avec ceux de sa complexifiée $\tilde{h}$. Pour cela on va introduire le $L$-type d'une fonction harmonique.

L'espace $\mathbf{R}^{N}$ étant muni de la norme euclidienne $x \mapsto\|x\|=\left(x_{1}^{2}+\cdots+\right.$ $\left.x_{N}^{2}\right)^{1 / 2}$, l'ordre $\rho$ et le type (euclidien) $\tau$ d'une fonction harmonique $h$ dans tout $\mathbf{R}^{\mathbf{N}}$ sont définis par analogie avec les fonctions entières dans $\mathbf{C}^{N}$ :

$$
\begin{array}{cl}
\rho(h)=\limsup _{r \rightarrow \infty}[\log \log m(h, r) / \log r], & m(h, r)=\max _{\|x\|=r}|h(x)| \\
\tau(h)=\limsup _{r \rightarrow \infty}\left[\log m(h, r) / r^{\rho(h)}\right] & \text { si } 0<\rho(h)<\infty .
\end{array}
$$

Rappelons que la norme de Lie dans $\mathbf{C}^{N}$ est l'application $L: \mathbf{C}^{N} \rightarrow \mathbf{R}_{+}$ définie par

$$
L(z)=\left[\|z\|^{2}+\sqrt{\|z\|^{4}-\left|\sum_{j=1}^{N} z_{j}^{2}\right|^{2}}\right]^{\frac{1}{2}} \quad\left(\|z\|^{2}=\sum_{j=1}^{N} z_{j} \bar{z}_{j}\right)
$$

et que $\operatorname{BL}(0, R)=\left\{z \in \mathbf{C}^{N} \mid L(z)<R\right\}$ est la boule de Lie dans $\mathbf{C}^{N}$ de centre 0 et de rayon $R$. La frontière de Bergman-Šilov de l'adhérence $\overline{\mathrm{BL}(0, R)}$ de $\mathrm{BL}(0, R)$ est l'ensemble $\check{\mathcal{B}}_{L}=\left\{x e^{i \theta} \mid\|x\|=R, x \in \mathbf{R}^{N}, \theta \in \mathbf{R}\right\}$. La boule de Lie est aussi la cellule d'harmonicité de la boule euclidienne $B(0, R) \subset R^{N}$ (cf. [2] p. 111). 
4.4. Définition. Soit $h$ une fonction harmonique dans tout $\mathbf{R}^{N}$ d'ordre fini $\rho(h)$ et de type $\tau(h)$. Si $\tilde{h}$ est le prolongement holomorphe de $h$ dans tout $\mathbf{C}^{N}$, on pose $m_{L}(\tilde{h}, r)=\max _{L(z) \leq r}|\tilde{h}(z)|$. On appellera $L$-type de $\tilde{h}$ le nombre

$$
\tau_{L}(\tilde{h})=\limsup _{r \rightarrow \infty} \frac{\log m_{L}(\tilde{h}, r)}{r^{\rho(\tilde{h})}} .
$$

4.5. Théorème. Soit $h$ une fonction harmonique dans tout $\mathbf{R}^{N}$ d'ordre fini $\rho(h)>0$ et de type $\tau(h)$. On a

$$
\rho(\tilde{h})=\rho(h) \quad \text { et } \quad \tau_{L}(\tilde{h})=\tau(h) .
$$

En particulier $|h(x)| \leq a \exp (b\|x\|)\left(x \in \mathbf{R}^{N}, a, b=\right.$ Cte $\left.>0\right)$ implique pour tout $\varepsilon>0$ l'existence d'une constante $A_{\varepsilon}$ telle que:

$$
|\tilde{h}(z)| \leq A_{\varepsilon} \exp [(b+\varepsilon) L(z)] \leq A_{\varepsilon} \exp [(b+\varepsilon)|z|] \quad\left(z \in \mathbf{C}^{N}\right)
$$

Remarquons que l'inégalité

$$
\|z\|^{4}-\left|\sum_{j=1}^{N} z_{j}^{2}\right|^{2} \leq 4 \sum_{i<k}\left|z_{j}\right|^{2}\left|z_{k}\right|^{2}
$$

implique $L(z) \leq|z|$ et que pour $z=x$ réel, $L(x)=\|x\|$. L'inégalité (32) améliore un résultat antérieur ( $c f$. [2], p. 157, Proposition 6.2.8).

4.6. Proposition. Soient $h$ une fonction harmonique dans la boule $B(0, R)$ $\subset \mathbf{R}^{N}$ continue sur son adhérence, $\mathrm{BL}(0, r)$ la boule de Lie de centre 0 et de rayon $r<R$ dans $\mathbf{C}^{N}$ et $\tilde{h}$ le prolongement holomorphe de $h$ dans $\mathrm{BL}(0, R)$. On a :

$$
m(h, r) \leq m_{L}(\tilde{h}, r) \leq \frac{1+(r / R)^{2}}{(1-r / R)^{N}} m(h, R) .
$$

Démonstration. En utilisant l'intégrale de Poisson de $B(0, R)$ on déduit l'expression de $\tilde{h}(z)$ dans la cellule d'harmonicité $\operatorname{BL}(0, R)$ :

$$
\tilde{h}(z)=\frac{R^{N-1}}{\sigma_{N}(1) R} \int_{\|\alpha\|=1} \frac{R^{2}-\left(z_{1}^{2}+\cdots+z_{N}^{2}\right)}{\left[\left(z_{1}-R \alpha_{1}\right)^{2}+\cdots+\left(z_{N}-R \alpha_{N}\right)^{2}\right]^{N / 2}} h(R \alpha) d \sigma(\alpha) .
$$

$(L(z)<R)$ on choisit la branche holomorphe du dénominateur qui est positive pour $z=x$ réel ; $\sigma_{N}(1)$ est la mesure-aire de la frontière de $B(0,1)$ et $\alpha \in \mathbf{R}^{N}$. En particulier $\tilde{h}(z)$ est holomorphe dans $\mathrm{BL}(0, r)(r<R)$. Le maximum de $|\tilde{h}(z)|$ 
dans $\overline{\mathrm{BL}(0, r)}$ est atteint sur la frontière de Bergman- $\breve{S i l o v} \check{\mathcal{B}}$, en un point $z_{0}$. Donc il existe $\stackrel{\circ}{x} \in \mathbf{R}^{N},\|\stackrel{\circ}{x}\|=r$ et $\theta_{0} \in \mathbf{R}$ tels que

$$
m_{L}(\tilde{h}, r)=\frac{R^{N-2}}{\sigma_{N}(1)}\left|\int_{|\alpha|=1} \frac{\left(R^{2}-r^{2} e^{2 i \theta_{0}}\right) h(R \alpha)}{\left[r^{2} e^{2 i \theta_{0}}+R^{2}-2 R e^{i \theta_{0}}\langle\dot{0}, \alpha\rangle\right]^{N / 2}} d \sigma(\alpha)\right|
$$

où $\langle\stackrel{\circ}{x}, \alpha\rangle=\|\stackrel{\circ}{x}\| \cdot\|\alpha\| \cos \omega=r \cos \omega$. En posant $a=(r / R) e^{i \theta_{0}}$ on obtient

$$
\begin{aligned}
m_{L}(\tilde{h}, r) & =\frac{\left|1-a^{2}\right|}{\sigma_{N}(1)}\left|\int_{|\alpha|=1} \frac{h(R \alpha) d \sigma(\alpha)}{\left[a^{2}-2 a \cos \omega+1\right]^{N / 2}}\right| \\
& \leq\left|1-a^{2}\right| m(h, R) \frac{1}{\sigma_{N}(1)} \int_{|\alpha|=1} \frac{d \sigma(\alpha)}{\left(\left|a-e^{i \omega}\right|\left|a-e^{-i \omega}\right|\right)^{N / 2}}
\end{aligned}
$$

mais $\left|a-e^{i \omega}\right| \cdot\left|a-e^{-i \omega}\right| \geq|| a|-1|^{2}$. D'où les inégalités (33) en remarquant que $B(0, r) \subset \mathrm{BL}(0, r)$ et que la restriction de $\tilde{h}$ à $\mathbf{R}^{N}$ est égale à $h$. Pour établir $\rho(h)=\rho(\tilde{h})$ on applique les inégalités (33) en choisissant tout d'abord $R=R(r)>r$ tel que $\log R(r) / \log r \rightarrow 1, r / R \rightarrow 0$ si $r \rightarrow \infty$. Ensuite pour établir $\tau(h)=\tau_{L}(\tilde{h})$ on choisira dans $(33) R=R(r)=[1+\varepsilon(r)]$ avec $r^{-\rho(h)} \log \varepsilon(r) \rightarrow$ $0, \varepsilon(r) \rightarrow 0$, si $r \rightarrow \infty$.

Corollaire. Si $h$ est harmonique, $|h| \leq M$ dans $B(0, R) \subset \mathbf{R}^{N}$, sa complexifiée $\tilde{h}$ vérifie en tout point de $\mathrm{BL}(0, R)$ l'inégalité

$$
|\tilde{h}(z)| \leq \frac{R^{2}+L^{2}(z)}{[R-L(z)]^{N}} R^{N-2} M \leq \frac{R^{2}+|z|^{2}}{(R-|z|)^{N}} R^{N-2} M .
$$

L'inégalité (34) complète un résultat de P. Lelong ( $c f$. [8] lemme 3.22, ou [2], 6.2.2).

Pour établir le corollaire, soit $z \in \mathrm{BL}(0, R)$. On a $r=L(z)<R$ et $|\tilde{h}(z)| \leq$ $m_{L}(\tilde{h}, r)$ (principe de maximum). D'où (34), d'après (33).

Remarquons que l'inégalité $L(z) \leq|z|$ et la croissance de la fonction $r \mapsto$ $\left(R^{2}+r^{2}\right) /(R-r)^{N}$ implique

$$
\frac{R^{2}+L^{2}(z)}{[R-L(z)]^{N}} \leq \frac{R^{2}+|z|^{2}}{\left(R-|z|^{N}\right)}
$$

L'inégalité (32) résulte de la définition de $\tau_{L}(\tilde{h})=\tau(h) \leq b$. 
4.7. Théorème. Soit $f$ une fonction $p$-harmonique $(p \geq 2)$, bornée dans la boule $B(0, R) \subset \mathbf{R}^{\mathbf{N}}$ et $\tilde{f}$ son prolongement holomorphe dans la cellule d'harmonicité $\mathrm{BL}(0, R)$. Alors en tout point $z \in \mathrm{BL}(0, R)$ on a :

$$
|\tilde{f}(z)| \leq A(N, p) r^{N-1} \operatorname{ch} \frac{r}{R-r} \frac{r^{2}+L^{2}(z)}{[r-L(z)]^{N+1}} \sup _{\|x\| \leq R} \mid(f(x) \mid,
$$

$A(N, p)=N(N+2) \cdots(N+2 p-4)(2 p-2)^{2 p}, L(z)<r<R$.

En particulier si $f$ est $p$-harmonique dans tout $\mathbf{R}^{N}$ et vérifie

$$
|f(x)| \leq k \exp (b\|x\|) \quad\left(x \in \mathbf{R}^{N}, k, b \text { ctes }>0\right)
$$

alors pour tout $\varepsilon>0$ il existe une constante $K_{\varepsilon}=K_{\varepsilon}(N, p, k, b)$ telle que

$$
|\tilde{f}(z)| \leq K_{\varepsilon} \exp [(b+\varepsilon) L(z)] \leq K_{\varepsilon} \exp [(b+\varepsilon)|z|] .
$$

Introduisons la fonction

$$
H_{f}(x, t)=\sum_{j=0}^{\infty}(-1)^{j} \frac{\Delta^{j} f(x)}{(2 j) !} t^{2 j} \quad\left(\Delta^{0} f=f\right)
$$

qui est harmonique pour $(x, t) \in B(0, R) \times R$ avec sa restriction à $t=0$ égale à $f(c f .[2], 1.1 .7)$.

4.8. Lemme. Avec les notations de 4.7 on a si $p \geq 2$ :

$$
\left|H_{f}(x, t)\right| \leq M A(N, p) \operatorname{ch} \frac{t}{R-r}, \quad(\|x\| \leq r<R, t \in \mathbf{R})
$$

Démonstration. On utilise le résultat suivant ( $c f .[2]$, p. 30) : Si $f$ est $p$ harmonique $(p \geq 2)$ bornée dans un domaine $\Omega \subset \mathbf{R}^{N}$ on a en tout point $x \in \Omega$ à distance $\delta(x)$ du bord de $\Omega$ les majorations :

$$
\left|\Delta^{q} f(x)\right| \leq A(N, p) \sup _{\Omega}|f| \delta^{-2 q}(x) \quad(q=0,1, \ldots, p-1) .
$$

Choisissons pour $\Omega$ la boule $B(0, R)$. Pour tout $x,\|x\| \leq r<R$ on a : $\delta(x) \geq R-r$. Donc, d'après (38) et (40):

$$
\left|H_{f}(x, t)\right| \leq A(N, p) \sup _{\Omega}|f| \sum_{j=0}^{\infty} \frac{1}{(2 j) !}\left(\frac{t}{R-r}\right)^{2 j}=M A(N, p) c h \frac{t}{R-r} .
$$

D'où (39). 
Revenons à la démonstration de 4.7. La fonction $H_{f}(x, t)$ est en particulier harmonique dans la boule

$$
\left\{(x, t) \in \mathbf{R}^{N} \times\left.\mathbf{R}\left|\|x\|^{2}+\right| t\right|^{2}<r^{2}\right\} \subset \mathbf{R}^{N+1}
$$

donc son prolongement holomorphe $\widetilde{H}_{f}(z, \zeta)$ dans la boule de Lie

$$
\left\{(z, \zeta) \in \mathbf{C}^{N+1} \mid L_{1}(z, \zeta)<r\right\} \subset \mathbf{C}^{N+1}
$$

où

$$
L_{1}(z, \zeta)=\left[\|z\|^{2}+|\zeta|^{2}+\sqrt{\left(\|z\|^{2}+|\zeta|^{2}\right)^{2}-\left|\left(\sum_{j=1}^{N} z_{j}^{2}\right)+\zeta^{2}\right|^{2}}\right]^{\frac{1}{2}}
$$

vérifie d'après (34) et (39) l'inégalité :

$$
\left|\widetilde{H}_{f}(z, \zeta)\right| \leq A(N, p) \sup _{\Omega}|f| c h \frac{r}{R-r} r^{N-1} \frac{r^{2}+L_{1}^{2}(z, \zeta)}{\left[r-L_{1}(z, \zeta)\right]^{N+1}},
$$

pour $L_{1}(z, \zeta)<r$. D'où $(35)$ en remarquant que $\tilde{f}(z)=\widetilde{H}_{f}(z, 0)$ et $L_{1}(z, 0)=$ $L(z)$.

Supposons que $f$ soit $p$-harmonique dans tout $\mathbf{R}^{N}$ et qu'elle vérifie (36) :

4.9. Lemme. Si $f$ est $p$-harmonique dans tout $\mathbf{R}^{N}$ et vérifie

$$
|f(x)| \leq k e^{b\|x\|} \quad(k, b \text { constantes }>0)
$$

alors pour tout $\varepsilon>0$, on a:

$$
\left|H_{f}(x, t)\right| \leq k A(N, p) \operatorname{ch} \frac{b}{\varepsilon} \exp [(b+\varepsilon)\|(x, t)\|]
$$

où $A$ est une constante ne dépendant pas de $\varepsilon$, et $\|(x, t)\|=\sqrt{\|x\|^{2}+t^{2}}$.

Démonstration. Pour $(x, t)$ donné $t \neq 0$, considérons dans $\mathbf{R}^{N}$ la boule $\Omega_{\varepsilon}$ de centre 0 et de rayon $\|x\|+\varepsilon|t| / b$. D'après (40) et du fait que la distance du point $x$ au bord de $\Omega_{\varepsilon}$ est $\varepsilon|t| / b$ on a:

$$
\left|\Delta^{q} f(x)\right| \leq A(N, p) \sup _{\Omega_{e}}|f|\left(\frac{b}{\varepsilon|t|}\right)^{2 q} \quad(q=0,1, \ldots p-1)
$$

et

$$
\begin{aligned}
\left|H_{f}(x, t)\right| & \leq A(N, p) \sup _{\Omega_{\varepsilon}}|f| \sum_{q=0}^{p-1}\left(\frac{b}{\varepsilon|t|}\right)^{2 q} t^{2 q} \\
& \leq A(N, p) k \exp b\left[\|x\|+\frac{\varepsilon}{b}|t|\right] \operatorname{ch} \frac{b}{\varepsilon} .
\end{aligned}
$$


En remarquant que $b\|x\|+\varepsilon|t| \leq(b+\varepsilon)\|(x, t)\|$ on obtient

$$
\left|H_{f}(x, t)\right| \leq k A(N, p) \operatorname{ch} \frac{b}{\varepsilon} \exp b\|(x, t)\| \quad(x, t) \in \mathbf{R}^{N} \times \mathbf{R} .
$$

Revenons à (37). Elle résulte de (32) et (42), en rappelant que

$$
\tilde{f}(z)=\widetilde{H}_{f}(z, 0), \quad f(x)=\tilde{f}(x) \quad(x \text { réel }) .
$$

4.10. Remarque. Si $f$ est une fonction $p$-harmonique dans tout $\mathbf{R}^{N}$, considérons la fonction $g_{f}: \mathbf{R}^{N} \times \mathbf{R}_{+} \rightarrow \mathbf{R}$ :

$$
g_{f}(x, t)=\sum_{j=0}^{p-1} \frac{\Delta^{j} f(x)}{j !} t^{j} \quad\left(\Delta^{0} f=f, t>0\right) .
$$

La fonction $g_{f}$ vérifie l'équation de chaleur :

$$
\left\{\begin{array}{l}
\left(\partial g_{f} / \partial t\right)(x, t)=\Delta_{x} g_{f}(x, t) \\
g_{f}(x, 0)=f(x)
\end{array}\right.
$$

Il est bien connu que la solution de (41) s'écrit :

$$
g_{f}(x, t)=\frac{1}{\pi^{N / 2}} \int_{\mathbf{R}^{N}} \exp \left[-\frac{\sum_{j=1}^{N}\left(x_{j}-\xi_{j}\right)^{2}}{t}\right] t^{-N / 2} f(\xi) d \xi
$$

En faisant le changement de variable $\xi_{j}+u_{j} \sqrt{t}$ on obtient

$$
g_{f}(x, t)=\frac{1}{\pi^{N / 2}} \int_{\mathbf{R}^{N}} \exp \left(-\|u\|^{2}\right) f(x+u \sqrt{t}) d u .
$$

Si $|f|$ est borné, (42) montre par $g_{f}$ l'est aussi. Comme celle-ci est un polynôme en $t$ on en déduit que $g_{f}(x, t)$ et par conséquent $g_{f}(x, 0)=f(x)$ est une constante. On retrouve ainsi une proposition bien connue (cf. [2], p. 30) : une fonction $p$-harmonique bornée dans $\mathbf{R}^{N}$ se réduit à une constante.

4.11. Proposition. Si $f$ est une fonction $p$-harmonique dans tout $\mathbf{R}^{N}$ telle que

$$
|f(x)| \leq A \exp (B\|x\|), \quad|f(\nu)| \leq M \quad\left(\nu \in \mathbf{Z}^{N}\right)
$$

où $A$ et $B, M$ sont des constantes positives et $B<\pi$. Alors $f$ est une constante.

L'exemple $f(x)=e^{\pi x_{1}} \cdot \sin \pi x_{2}$ dans $\mathbf{R}^{2}$ montre que l'hypothèse $B<\pi$ est la meilleure possible. 
La démonstration résulte de l'inégalité (37) et le théorème Cartwright pour plusieurs variables ( $c f$. [10], p. 40) : Puisque la complexifiée $\tilde{f}$ de $f$ vérifie (37) et (43), il existe une constante $L>0$ (d'après le théorème de Cartwright) telle que $|f(x)| \leq M L\left(x \in \mathbf{R}^{N}\right)$. Donc $f=$ constante d'après 4.10 .

\section{Références}

[1] Avanissian, V., et R. Gay: Sur une transformation des fonctionnelles analytiques. - Bull. Soc. Math. France 103, 1975, 341-384.

[2] Avanissian, V.: Cellule d'harmonicité et prolongement analytique complexe. - Travaux en Cours, Hermann, Paris, 1985, 1-183.

[3] Avanissian, V.: Sur les fonctions $p$-harmoniques de type exponentiel. - C. R. Acad. Sci. Paris 304, Sér. I 16, 471-474, 1987.

[4] Avanissian, V.: Sur le $L$-type du prolongement holomorphe des fonctions harmoniques entières. - C. R. Acad. Sci. Paris 307, Sér. I, 1988, 149-152.

[5] Avanissian, V.: Fonctionnelles analytiques liées aux polynômes orthogonaux classiques. - C. R. Acad. Sci. Paris 307, 1988, 177-180.

[6] GAY, R.: Division des fonctionnelles analytiques. - Thèse, Université Louis Pasteur, Département de Mathématiques, Strasbourg, 1976.

[7] Lebedev, N.N.: Special functions and their appications. - Dover Publications, Inc., 1972.

[8] Lelong, P., et L. Graman: Entire functions of several complex variables. - Grundlehren der mathematischen Wissenschaften 282. Springer-Verlag, 1986, 178-200.

[9] Martineau, A.: Sur les fonctionnelles analytiques et la transformation de Fourier-Borel. - J. Analyse Math. II, 1963, 1-164.

[10] Yoshino, K.: Some examples of analytic functionals and their transformation. - Tokyo J. Math. 5, 1982, 479-490.

Université Louis Pasteur

Institut de Recherche Mathématique Avancée

10, rue du Général Zimmer

F-67084 Strasbourg Cedex

France

Received 7 March 1989 